\title{
A Link Between Colors and Emotions; A Study of Undergraduate Females
}

\author{
Achint Kaur \\ MCM DAV College, Panjab University, Chandigarh, India
}

\begin{abstract}
This study examined the association of 3 primary colors blue, red and yellow (Munsell color system) and 3 neutral colors: black, white and grey to 4 emotions: happiness, fear, anger and sadness, in females 18-21 years old. The undergraduate females from various background, courses and age were required to associate the colors to the specific emotions and state why. It was predicted that the three primary colors would be associated with high energy emotions such as anger and happiness and the three neutral colors with low energy emotions such as sadness and fear in the sample. In the results, more than half of the subjects associated the primary colors with happiness, anger. Black, was associated majorly with happiness, red with fear, grey to sadness, White with fear. The color blue was poorly linked with anger and strongly with happiness like the color yellow across all the age groups.
\end{abstract}

Keywords:- Color, fear, anger, black, neutral colors.

\section{INTRODUCTION}

Humans have a keen tendency to associate colors with emotions. This is prevalent in large number of people, from all walks of life, across the globe. Neutral colors such as black and white, often have important significance in religious moments and are used as a medium of expression in ceremonies and daily life. For example, wearing black on funeral in western countries, such as United States of America, is an old tradition. Furthermore, the color white, worn on weddings by brides, holds a totally different meaning on why its worn, in concise the expression is different.

In one study conduct on 40 undergraduate college students, 20 being males and 20 females, it was observed that bright colors had positive emotional response linking and dark colors with negative emotional linking. The females reacted more positively to bright colors and more negatively to dark colors (Hemphill, 1996). It was also concluded that colors have strong impact on our emotions and feelings (Hemphill, 1996). For example, red color is associated with excitement, orange with unhappiness and violet with dignity (Ballast, 2002). It has also been observed that a single color can be associated with multiple emotions (Satio, 1996; Linton 1999). In another research, it was observed that the various colors worn in the form of clothes, had varied impact on the emotional states of primary school children aged 7-11. Like, for the students of 3rd grade, the color red leads them to feel confusion and indifference (Parac-Osterman et al., 2011). Red as a color is dynamic, it stimulates excitement. This color has positive and negative emotional impact. The color green has relaxing and soothing effect, with links to feeling fatigue as well (Satio 1996; Davey 1998; Mahnke 1993)

Through a couple of studies, it was proven that some emotions are closely associated with some colors regardless of age, sex, culture and nationality. In yet another study it was observed that color preferences can be cultured based, the study was done by Choungourian(1968) and it was found that red and blue were more preferred amongst Americans than other cultures. In another research by Boyatzis and Varghese (1994), it was observed that light colors are linked to positive emotions and dark colors with negative emotions. In the study by Hemphill (1996) it was observed that pure hues of colors are associated with positive emotional responses and dark hue with negative ones. But, by Satio (1996) it was seen that dark colors have positive savors as well, in the Japanese population.

Colors can be described as warm or cold colors. The example of warm colors are orange and red and cold colors are blue and green. The warm colors are known to be of high energy and that they stimulate energy while cold colors are relaxing (Ballast, 2002). Cold colors are known to make a place feel enlarged and spacious, while the warm color make it feel small and constricted (Lang, 1993). People have reported to have higher level of anxiety when exposed warmer colors than cold colors like blue and green. (Kwallek, Lewis, \& Robbins, 1988; Mahnke \& Mahnke, 1993). In a study conducted on 98 undergraduate students it was observed that the principal hues had the largest number of positive emotional response, with the color yellow and green having lowest number of positive responses as it was associated with vomit and sickness. For the neutral color, white received highest number of positive responses (Kaya et al., 2004)

There are multiple color systems that exist (Parac-Osterman , 2004 see for discussion) and can be used. The best one is Munsell color system and it classifies colors on the basis of hue, Chroma and lightness. The total number of hues are 10 and 5 of them are main hues (e.g.: red, yellow). According to this system, black, white and grey are called as neutral colors. Chroma is the degree of color saturation with the main hue. Hue helps us distinguish one color from another. Value is an important aspect of color and is the degree of lightness and darkness.

\section{Participants}

\section{METHODOLOGY}

The sample collected, consisted of 141 undergraduate females from various colleges across Chandigarh, India. They were all willing volunteers who were from Bachelor of Arts, Bachelor of Science and Bachelor of business administration branches. All the students were from various years of their degrees. None of the females were color blind and were also not diagnosed with 
any prior psychological disorders. Some females wore spectacles and had 6/6 vision with it, which they had confirmed from their doctors. Some potential candidates were removed as they were on hallucinogens drugs and drinks. The participants were from the age group of $18-21$, out which $36 \%$ were 19 years old, $36.7 \%$ were 18 years old, $15.8 \%$ were 20 years old and $11.5 \%$ were 21 years old. 18 Was their average age.

\section{MATERIAL}

All the female participants were required to take an online color-blind test from https://enchroma.com before answering the prepared, online questionnaire. The students were then provided with an online questionnaire which showed a single color and were asked questions which were: which of the following color would you associate with this emotion? Why did you select this color for this emotion? With additional questions; Would you associate this color with happy or angry moments and events in your life? and would you associate this color to sad/pessimistic/unhappy events and moments in your life?. These questions were adapted from Boyatzis and Varghese (1994), Hemphill (1996). In the questionnaire, they were provided with the pictures $(9 \mathrm{~cm}$ $11 \mathrm{~cm}$ ) of the colors on a white background, the colors were from Munsell color system. The colors were: red(10R 3/10), blue( 10BG 1/2), white, black, yellow(7.5Y 9/10 )and grey(10YR 3/2). There was no time limit set on the questionnaire.

\section{PROCEDURE}

Data was studied by using Statistical Package for Social Sciences ( SPSS) along with using descriptive statistics. All the female participants were provided with the link to the questionnaire through online media platforms such as WhatsApp, Facebook and Instagram, along with email, on their devices of choice. Before the conduction of the questionnaire they were required to conduct a test on themselves, with the link to an online color-blindness testing site namely: https://enchroma.com , which measures the ability to see a pattern based on its color. It tests with various colors, to see which colors the participants had a problem with. In the test, they viewed a series of images composed of dots with varying size and lightness. The participants were then suppose to look for a shape, such as a number or symbol, which was embedded within the dot pattern. The time limit for each image was 5 seconds and there were 9 color plates in all, taken from Ishihara color plates. In the beginning, the participants gave an online consent to be part of the research. In the instructions given before the questionnaire, they were required to settle themselves comfortably in a location where they cannot be disturbed by other family members, friends, instruments, appliances and pets etc, to conduct the questionnaire. This was done to reduce distractions, which effects the decision making in humans (Li et al., 2018). The questionnaire was predicted to take almost 5 minutes to complete, and there was no time limit set for completing the questionnaire. This was done specifically to ensure that students don't attempt questions in a hurry, give proper time to all question and carefully evaluate the options. The participants were asked to state their age and if they had been diagnosed with any prior psychological disorder, those who were, their answers were not considered for the test. The names of the students weren't exposed or announced in the results due to privacy issues. There were multiple choice questions in the questionnaire, that asked to link each of the 6 colors with one emotion from the given options and also state their reasons for why.

\section{RESULTS}

To study the data, we used Statistical package for Social Sciences (SPSS) soft-ware program and descriptive statistics were used to compare the data. Based on the responses from the females it was observed that $36 \%$ were 19 years old and 18 years old accounted for $36.7 \%$. 21 years old females were only $11.5 \%$. Our hypothesis stated that the three primary colors would be associated with high energy emotions such as anger and happiness and the three neutral colors with low energy emotions such as sadness and fear, in all age groups. According to the data, it was observed that, $57.4 \%$ students did not associate either of the colors black, white and grey with sad/ pessimistic/unhappy/fearful moments in their lives, reason being they found the color white as stylish, peaceful and color black as peppy and grey was associated with grace. For this, out of the total respondents, $42.55 \%$ were 19 years old, who refused to associate the neutral colors with such emotions and $37.5 \%$ out of 16,21 years old gave a positive answer to the question, stating the reason that they associated the colors with death and darkness, death being the major categories of choice. Also, for this $40 \%$ of the total 18 years and $40 \%$ of 19 years old respondents gave yes as an answer. It can be concluded from the data collected, that 19 and 18 years old females are slightly more susceptible to linking unhappy events and emotions to neutral colors more than 21 years old. But, the highest number of age group that linked the neutral colors to sad/pessimistic /unhappy events and moments was 20 years old, with $47.6 \%$ voting for yes. It can be said that there is no particular pattern across the age groups that could be found, where the students associate grey, white and black with sad, fearful etc. moments and emotions such as sadness and fear.

$55.6 \%$ females associated the colors blue, red and yellow, with blue being in the majority with happy/joyful/fun/angry moments and memories, because for them blue radiated vogue, style, power and charisma, with style being the majority's choice. In this 13 out of 16, 21 years old associated the primary colors with happy, chirpy etc. moments in their lives, this is twice more than the positive response they gave to the linking of the 3 neutral colors with negative and pessimistic events and moments of their lives. $36 \%$ of 18 years old answered it negatively along with $46 \%$ of all 19 years old as well. Hence, it can be concluded by reviewing the data, with the statement that 19 years old are less likely to link the primary colors with happy and angry events and moments of their lives than 21 and 18 years old and in an estimate, it can be more than $30 \%$ less likely to do so. More than $60 \%$ of the 20 years old disagree as well. there is an uneven pattern of connecting primary colors to joyful/angry/happy emotions across the 4 age groups. The 20 years old students have close values of positive linking in terms of the two previously talked about associations. By using pie charts on the data collected, it was observed that $53.2 \%$ of the females felt that the color red 
makes them feel angry, which is followed by $19 \%$ people feeling yellow makes them angry, this is followed by grey, blue, black and white (in the same order). This was because more than $60 \%$ felt were reminded of danger, sign boards, police and fire by the color red, with sign boards being a popular choice and the color yellow reminded them of vomit, feces, heat and blasts. In this, less than 8 participants (less than 50\%) who were of the age 20 found that the color red makes them feel angry and $14.28 \%$ associated anger with the neutral color grey. Only four students from both 18 and 19 years old category associated black color with anger. It can be concluded that from the age group 18 to 21 there is a small increase in the preference of association of neutral colors to anger. More than $66 \%$ of the collected and tested students, across the 4 age groups associate the three primary colors red, blue and yellow to anger, with the color red winning the race. The age group which associated maximum, the neutral color like black to anger was 21 .

None of the participating females had been diagnosed with any color blindness issues and psychological disorders. Furthermore, upon reviewing the results it was observed that the color black majorly linked with the emotion happiness by $27.3 \%$ participants which was closely followed by yellow and then blue. This was because they associated black with energetic moments, power, peppy, style and grace. The color yellow here reminded them of flowers, happiness and rainbows, with rainbows being the popular choice. The pattern of associating black with the emotion happiness showed a steady decline, when we descend from the age 21 to 18 years, with only 12 people associating it with happiness in the age group of 18 . It can be said, from the data collected that as females progress through their lives they stop associating neutral colors with happiness and associate primary colors more with it from the age group of 18 to 21. Moreover, it was seen through the pie charts and other statistical graphs that nearly $36 \%$ students associated the color red with fear which was closely followed by black, at $25.4 \%$ and a tie between the colors grey and white was observed. This was due to the fact that red was associated here with blood, pain and hell, with blood being the majority's opinion. It was concluded that there was no pattern that came out for this association across the years. With $30 \%$ of 19 years old females opting for red as a color that brings out fear, it was the highest across the 4 age groups which was followed by $25 \%$ of 21 years old. There was a $10 \%$ increment in the association of color black to fear from the age group 18 to 20 and it was followed by a sharp decline of $13.9 \%$ in the age group of 21 . This signals to an uneven pattern of associating the color black with fear from 18 to 21 age group, with the decline present in the age groups of 19 and 21 . Here the students associated black with chaos, destruction, malice etc.

Furthermore, in the research it was observed that $47.6 \%$ of the participants associated the color grey with the emotion sadness with majority of them being 18 years old. This was because the females associated grey with depression, cloudy sky, sadness etc. This was followed by $14.8 \%$ for the color blue, after which came a tie between the colors white and yellow. It was concluded that 18 years old females were more likely to associate the color grey to sadness than 20 and 21 years old.

\section{DISCUSSION}

The main goal of this research was to establish the association between the primary and neutral colors with 4 emotions which are happiness, anger, fear and sadness and to discover the reasons why, across the females of age groups 18 to 21 , by using a Munsell color system, in which it was hypothesized that the primary colors( blue, red, yellow) would be associated with happiness and anger, and the three neutral colors with depressive emotions ; sadness and fear. It was observed that color red elicited the negative emotion fear more than the neutral color black and white and was closely followed by the color yellow. The reason for this was that the students associated the color red with danger, death, blood and hell and as for the color yellow, they associated it with vomit, blasts and heat, thus bringing in the element of war and disgust. There was more than 5\% increment in the association of color black to fear from the age group 18 to 20 and it was followed by a sharp decline of more than $10 \%$ in the age group of 21 . This signals to an uneven pattern of associating the color black with fear from 18 to 21 age group, with the decline present in the age groups of 19 and 21 . Here, the students associated black with chaos, destruction, malice etc.

The color black failed to elicit as much fear as color red and yellow, because it was considered as a stylish, peppy color that reminded the students of elegance. The emotion happiness was majorly associated with the color black by the students, reason being it provided the student the feeling of power, energy and they associated the color as 'peppy'. The color yellow came second in being associated with happiness and it reminded them of rainbows, sunshine and flowers. It was observed that there was a decline in associating color black with happiness as we travel from the age group 21 to 18 . As the females aged, they stopped associating colors black, white and grey with happiness and anger and primary colors more with it. The color blue failed to associate highly as compared to red and yellow to any of the 4 emotions and was associated with sky, water, style and charisma with sky being the majority. The color grey has been highlighted as eliciting very depressive emotion; sadness, by females of all age groups, with the 18 years old females associating it more with sadness than any other of the three groups and the least in the age groups of 21 . From the statistical graphs it was observed that the color red was eliciting anger and rage in more than $50 \%$ of the students as it reminded them of screams, danger, pain and sign boards in broad category. White was the least color the students associated with anger as it was perceived to be a color of purity and peace. Majority of the females associated the colors red, blue and yellow with positive/happy/angry events in their lives, with the numbers increasing from the age 21 to 18 . It was concluded that there were various pointers that helped females associate emotions to color, at times it was culture and at times it was personal preference and experience. The color white was highly linked with purity, peace and dullness as was in the case of Satio' study (1996). At times white was associated as a depressive color as it was considered bland and plain.

This research and similar, along with future research could highlight trends in associating colors to emotions across nations and cultures. This study could also be helpful in market research of which colors to use for better sales in terms of products eg: which colored product makes the customer feel sad or happy. Future studies on these lines can also help increase the understanding of 
which colors combinations are associated with which emotions. Further studies could be conducted on the association of color to emotions when the colors are presented on different colored background. For more studies they could also use rating scales for studying association of colors such as "hot and cold" etc. We can also study the association of color to emotion variation during hormonal changes in a female.

\section{REFERENCES}

[1] Adams F.M., C.E. Osgood: A crosscultural study of the affective meaning of colour. Journal of Cross-Cultural Psychology 7 (1973) $135-157$

[2] Ballast D.K.: Interior design reference manual, Professional Pub. JjmInc. (2002) Belmont, CA.

[3] Boyatzis, C. J., \& Varghese, R. (1994). Children's emotional associations with colors. Journal of

[4] Genetic Psychology, 155, 77-85.

[5] Choungourian, A. (1968). Color preference and cultural variation. Perceptual \& Motor Skills, 26, 1203-1206.

[6] Davey P.: True colours: The glorious polychromy of the past suggests a strong historical need for

[7] colour, despite current reductive fashions, The Architectural Review 204 (1998) 34-36

[8] Eysenck H.J.: A critical and experimental study of colour-preferences. American Journal of Psychology 54 (1941) 385-394

[9] Hemphill, M. (1996). A note on adults' color-emotion associations. Journal of Genetic Psychology,157, 275-281.

[10] Kaya, N., \& Epps, H. H. (2004). Relationship between color and emotion: A study of college students. College Student Journal, 38(3), 396-405.

[11] Kwallek. N., Lewis, C. M., \& Robbins, A. S.(1988). Effects of office interior color on workers' mood and productivity. Perceptual \& Motor Skills, 66, 123-128.

[12] Lang J.: Creating architectural theory: The role of the behavioral sciences in environmental design, New York(1993) Van Nostrand Reinhold

[13] Li, V., Michael, E., Balaguer, J., Herce Castañón, S. and Summerfield, C., 2018. Gain control explains the effect of distraction in human perceptual, cognitive, and economic decision making. Proceedings of the National Academy of Sciences, 115(38), pp.E8825-E8834.

[14] Linton H.: Colour in architecture: Design methods for buildings, interiors and urban spaces, New York (1999) McGraw Hill

[15] Mahnke F.H., R.H. Mahnke: Colour and light in man-made environments, New York (1993) Van

[16] Nostrand Reinhold

[17] Parac-Osterman, Đ., Hajsan-Dolinar,V., Ira Glogar, M.(2011) Clothing colours and behaviour of pupils of primary school age . Tekstil, 60 (7), 295. Saito, M. (1996). Comparative studies on color preference in Japan and other Asian regions, with special emphasis on the preference for white. Color Research and Application, 21(1),35-49.

\section{TABLE AND GRAPHS}

Munsell color chart.

\begin{tabular}{|l|l|l|}
\hline Color & Hue & Value/Chroma \\
\hline Red & $10 \mathrm{R}$ & $3 / 10$ \\
\hline Yellow & $7.5 \mathrm{Y}$ & $9 / 10$ \\
\hline Blue & $10 \mathrm{BG}$ & $1 / 2$ \\
\hline White & $\mathrm{N} / 9$ & \\
\hline Black & $\mathrm{N} / 1$ & \\
\hline Grey & $10 \mathrm{YR}$ & $3 / 2$ \\
\hline
\end{tabular}

\section{state your age}

\section{9 responses}

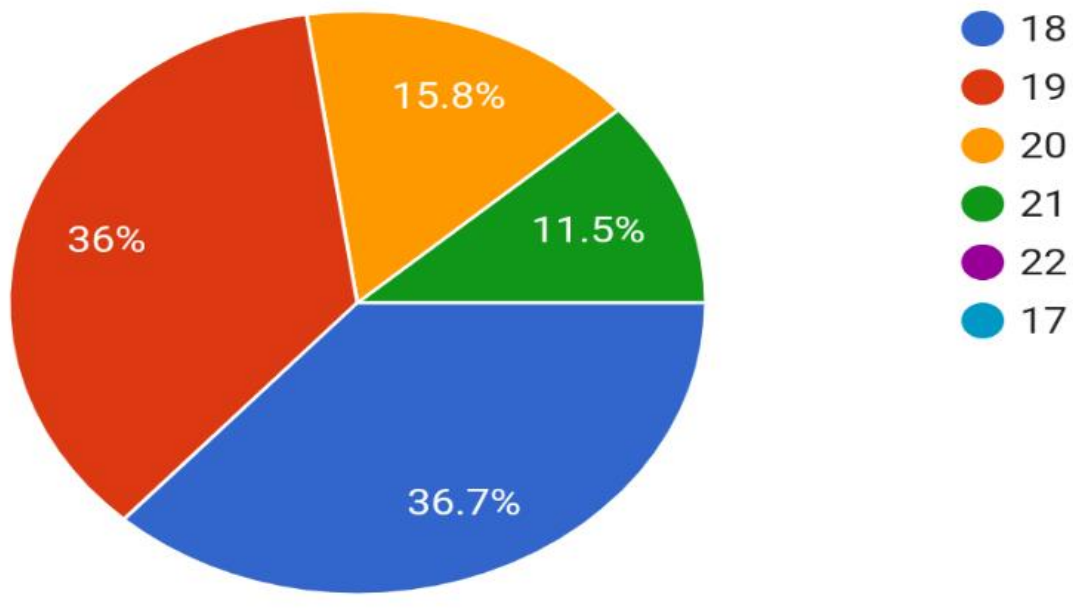


which of the following color makes you feel sad? WHY? Blue, red, yelow, white, grey, black

\section{2 responses}

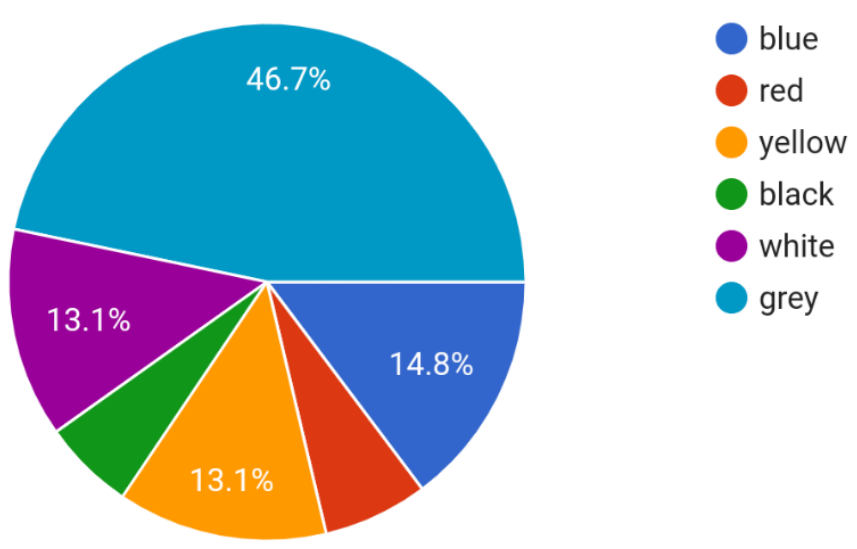

which of the following color makes you feel fearful? WHY? Blue,

Red, Yellow, Black, white, grey

126 responses

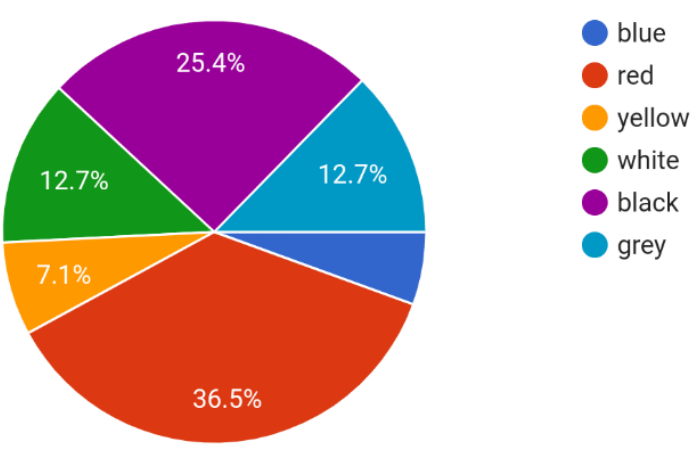

